Pierre Bouzat

Pierre J. Magistretti

Mauro Oddo

\section{Hypertonic lactate and the injured brain: facts and the potential for positive clinical implications}

Accepted: 17 April 2014

Published online: 1 May 2014

(C) Springer-Verlag Berlin Heidelberg and ESICM 2014

The letter referred to here can be found at doi:10.1007/s00134-014-3297-5.

Dear Editor,

We thank Nordström and colleagues for their interest in our study. We shall try to summarize their criticisms and to answer them point by point.

1. The statement that cerebral glucose increase following lactate infusion may be due to a shift in the cell redox state is debatable in the absence of NADH/NAD ratio measurement. Lactate can increase because of anaerobic glycolysis, due to lack of oxygen, but alsofrequently-when brain oxygen is normal [1]. In such conditions, exogenous lactate can then be converted to pyruvate, which is subsequently metabolized via the TCA cycle, a process known as aerobic glycolysis [2].

2. Lactate oxidation yields $32 \mathrm{~mol} \mathrm{ATP}$ vs. 34 mol ATP during glucose oxidation, a small difference. In contrast to glucose oxidation, lactate oxidation does not require ATP, which is an advantage in conditions of limited energy reserves.

3. Abundant evidence shows that exogenous supplemental lactate acts as a "glucose-sparing substrate". Semantic debate remains as to whether lactate might be described as a "preferential" or "opportunistic" substrate [3]. What is more relevant from the clinical standpoint is the potential benefit of sparing cerebral glucose: since low cerebral microdialysis (CMD) glucose is frequent after traumatic brain injury (TBI) and is associated with worse outcome [4], increasing CMD glucose during the early critical phase of TBI with lactate therapy clearly appears beneficial. Directly increasing glucose delivery to the brain with glucose infusions cannot be recommended because it might lead to hyperglycemia and hypotonicity, thereby exacerbating cerebral damage.

4. A positive correlation between CMD pyruvate and outcome has been described [4]; therefore the increase of brain pyruvate during lactate infusion seems beneficial. A pattern of elevated lactate-topyruvate ratio with normal to high pyruvate is not an exclusive hallmark of mitochondrial

dysfunction, because it is also observed during activated cerebral glycolysis.

5. Finally, using robust mixed-effects model statistical analysis we found that all CMD markers, including CMD glutamate, did not change in the $6 \mathrm{~h}$ previous to the intervention [5]. During lactate therapy CMD glutamate (that should have indeed been presented in $\mu \mathrm{mol} / \mathrm{L}$ instead of $\mathrm{mmol} / \mathrm{L}$ ) decreased almost significantly $(p=0.06)$. Since CMD glutamate might rise dramatically after TBI [4] - and the failure to remove this excess glutamate might lead to excitotoxic damage and cell deathproviding energy in the form of lactate, by increasing glucose availability, helps sustain glutamate uptake in astrocytes and allows neurons to prevent glutamate "leak".
6. The decrease of ICP following lactate infusion might indeed primarily be due to hypertonic sodium. Therapy of intracranial hypertension with hypertonic lactate solutions might therefore exert favorable anti-edematous and proenergetic effects and could be a valid therapeutic strategy after TBI.

In summary, we provided convincing data that exogenous supplemental lactate can be used as a preferential glucose-sparing substrate by the injured brain and that hypertonic lactate might improve neuroenergetics and reduce brain edema, thereby attenuating secondary cerebral damage after TBI. These data constitute robust arguments against all criticisms by Nordström and colleagues. More importantly, they support clinical investigation to examine the potential therapeutic advantages of hypertonic lactate solutions after brain injury.

Conflicts of interest None to declare.

\section{References}

1. Sala N, Suys T, Zerlauth JB, Bouzat P, Messerer M, Bloch J, Levivier M,

Magistretti PJ, Meuli R, Oddo M (2013) Cerebral extracellular lactate increase is predominantly nonischemic in patients with severe traumatic brain injury.

J Cereb Blood Flow Metab 33:1815-1822

2. Raichle ME, Mintun MA (2006) Brain work and brain imaging. Annu Rev Neurosci 29:449-476

3. Pellerin L, Magistretti PJ (2012) Sweet sixteen for ANLS. J Cereb Blood Flow Metab 32:1152-1166

4. Timofeev I, Carpenter KL, Nortje J, AlRawi PG, O'Connell MT, Czosnyka M, Smielewski P, Pickard JD, Menon DK, Kirkpatrick PJ, Gupta AK, Hutchinson PJ (2011) Cerebral extracellular chemistry and outcome following traumatic brain injury: a microdialysis study of 223 patients. Brain 134:484494 
5. Bouzat P, Sala N, Suys T, Zerlauth JB, M. Oddo

Marques-Vidal P, Feihl F, Bloch J,

Messerer M, Levivier M, Meuli R,

Magistretti PJ, Oddo M (2014) Cerebral metabolic effects of exogenous lactate supplementation on the injured human brain. Intensive Care Med 40:412-421. doi:10.1007/s00134-013-3203-6

P. Bouzat $(\bowtie) \cdot$ M. Oddo

Neuroscience Critical Care Research Unit, Department of Intensive Care Medicine, Centre Hospitalier Universitaire Vaudois (CHUV), University Hospital and Faculty of Biology and Medicine, Rue du Bugnon 46, 1011 Lausanne, Switzerland e-mail: PBouzat@chu-grenoble.fr e-mail: mauro.oddo@chuv.ch

P. Bouzat

Joseph Fourier University, Grenoble, France

P. J. Magistretti

Laboratory of Neuroenergetics and Cellular Dynamics, Centre de Neurosciences

Psychiatriques (CHUV), Brain Mind

Institute, Ecole Polytechnique Fédérale de

Lausanne (EPFL), University Hospital and

Faculty of Biology and Medicine,

1011 Lausanne, Switzerland
P. J. Magistretti

BESE Division, KAUST, Thuwal, Saudi Arabia 